\title{
Diseño y construcción de prototipos de heliodón para simulador de trayectorias solares
}

\author{
Design and construction of heliodon prototypes for solar trajectory simulator
}

Projeto e construção de protótipos de heliodono para simulador de trajetória solar

Thadee Birzavitt García Quintero.

Arquitecto.

Universidad Veracruzana, México.

thadebirzavittgarcia57@gmail.com

(iD) https://orcid.org/0000-0001-7701-7618

Carlos César Morales Guzmán.

Arquitecto, Ph.D. Arquitectura.

Universidad Veracruzana, México.

dr.arqmorales@gmail.com .

Recibido: agosto 2 de 2021

(D) http://orcid.org/0000-0002-4499-6968

\section{RESUMEN}

Se presenta el desarrollo y funcionamiento de dos modelos de simuladores de trayectorias solares (Heliodón) y su aplicación en la Arquitectura. Se observa el impacto de las sombras sobre una vivienda y la orientación correcta del proyecto utilizando estos prototipos. El proceso de diseño y construcción del Heliodón permite aprovechar al máximo la incidencia solar en la Arquitectura y otorgarle a las viviendas un menor consumo energético, a fin de disminuir la afectación al medio ambiente.

Palabras clave: Modelado; Heliodón; Prototipado; Experimentación.

\begin{abstract}
The development and operation of two models of solar trajectory simulators (Heliodon) and their application in Architecture are presented. Using these prototypes, the impact of shadows on a house and the correct orientation of the project is observed. The design and construction process of the Heliodon makes it possible to take maximum advantage of the solar incidence in the Architecture and to give the houses a lower energy consumption, in order to reduce the impact on the environment.
\end{abstract}

Keywords: Modeling; Heliodon; Prototyping; Experimentation. 


\section{RESUMO}

São apresentados o desenvolvimento e operação de dois modelos de simuladores de trajetória solar (Heliodon) e sua aplicação em Arquitetura. O impacto das sombras em uma casa e a orientação correta do projeto são observados usando esses protótipos. O processo de desenho e construção do Heliodon permite tirar o máximo partido da incidência solar na Arquitectura e dar às habitações um menor consumo de energia, de forma a reduzir o impacto no meio ambiente.

Palavras-chave: Modelagem; Heliodon; Prototipagem; Experimentação.

\section{INTRODUCCIÓN}

La incidencia solar sobre la superficie terrestre ha sido estudiada desde el surgimiento de la Arquitectura en todas las civilizaciones. Sin embargo, en el siglo XX esta práctica arquitectónica fue dejada de lado por muchos constructores y reemplazada por las superficies acristaladas en los edificios, y casi un siglo después se dieron cuenta de que esta arquitectura demanda un alto consumo energético al tener que generar confort térmico artificialmente en climas extremos, por lo que, a inicios del siglo XXI, se retomó este estudio y se le dio prioridad a la disminución de consumo energético, al considerarse como una de los aspectos que genera mayor afectación al ambiente (Galván, 2005).

Sin embargo, el enfoque energético en la Arquitectura solo es un elemento que forma parte del diseño bioclimático y sustentable, por lo que no es suficiente lograr un balance o confort térmico en las edificaciones porque se debe lograr una percepción agradable de la iluminación natural dentro de los espacios (Beckers, 2007).

En el diseño arquitectónico moderno se realiza un análisis de sitio del proyecto pero en la mayoría de los casos sólo se analizan datos teóricos donde se toman en cuenta estadísticas, pero no se visualiza a profundidad el comportamiento de estos factores, por lo que al momento de considerarlos en el diseño de una edificación los resultados no son completamente satisfactorios, es por eso que surge la necesidad en los procesos de formación en Arquitectura, la construcción de prototipos de Heliodón ya que estos permiten interpretar el impacto solar sobre un proyecto con gran detalle en cualquier hora, fecha y ubicación que se requiera (Gómez y Román, 2020).
El origen del surgimiento de la necesidad por saber la trayectoria solar y analizar su impacto sobre la Tierra es muy antiguo y abarca muchos temas. Por lo que nos enfocaremos en la necesidad del Arquitecto por conocer la trayectoria solar y su función dentro del diseño de un proyecto. El nombre de Heliodón proviene del griego: máquina solar. Es una útil herramienta en la etapa de diseño y permite por medio de ella estudiar las sombras, el acceso a la radiación solar de las construcciones y los niveles de iluminación natural.

La astronomía babilónica establece las bases y metodologías científicas para el estudio de esta ciencia; posteriormente, la antigua mesopotamia retomaría estos conocimientos para ampliar el campo de la astronomía a futuras civilizaciones como los griegos, sirios, musulmanes y europeos (Fragoso, 2020).

El arquitecto necesita un sistema de notación, que le permita jugar con la geometría y así revelar sus posibles relaciones con el Sol y el espacio tridimensional que ayude a evaluar las necesidades del usuario (Rodríguez, 2007).

El simulador solar permite una visión tridimensional del comportamiento del sol frente a los edificios, aunque implica la construcción de modelos o maquetas físicas a escala. Este dispositivo está compuesto principalmente de 3 elementos: 1. La base, que representa el plano donde se ubican las edificaciones. 2. El arco solar, que representa el recorrido de la trayectoria del Sol y 3. La lámpara, que representa el Sol en sí y proyecta una vista previa del impacto de luz solar sobre el proyecto. La clave para simular de manera correcta las sombras proyectadas por los volúmenes sobre el plano es controlar los ángulos de inclinación y declinación del arco solar y de la lámpara, así como la orientación del norte mediante una base 
giratoria, es decir, que todos los componentes del Heliodón sean móviles, pero que la base principal permanezca fija. Al diseñar un Heliodón, casi siempre implica la construcción de una fuente de luz artificial que simula la acción del sol. Esta fuente, fija o móvil, se relaciona con el resto de la instalación destinada a ubicar las tres variables de la geometría solar: la latitud (determina los ángulos de posición solar en relación con el lugar); el día del año (determina el ángulo de declinación solar en ese día); y la hora del día (determina los ángulos de posición solar para cada hora del día).

El Heliodón sirve como una herramienta para determinar con una proyección más específica la relación Sol-Edificio y es útil para visualizar una configuración real y eficaz de los elementos arquitectónicos (Tamiset, Tirado y Gámez, 2013). Los heliodones se suelen clasificar de manera general como: 1. De fuente luminosa fija y modelo arquitectónico móvil. 2. De fuente luminosa móvil y modelo fijo. Y 3. De fuente luminosa y modelo móvil (Maristany \& Barra, 2007).

En este artículo se presenta el proceso de diseño y construcción de 2 propuestas modelisticas de un Heliodón solar, dentro de un proceso de investigación que busca el desarrollo de estos dispositivos, como herramienta didáctica para el aprendizaje de conceptos bioclimáticos en la enseñanza de la Arquitectura e Ingeniería.

\section{MÉTODOS}

Primer Prototipo. A pesar de que la mayoría de heliodones existentes poseen una fuente de iluminación artificial, en este caso el primer prototipo experimentará con una fuente de iluminación natural: el Sol. Esto, con el fin de generar una alternativa simple y análoga de un dispositivo que, en lugar de recurrir a accesorios artificiales, utilice herramientas clásicas como la rosa de los vientos y la carta solar. Así pues, se propone este dispositivo configurado así:

Está compuesto de una base en forma circular que simula el plano horizontal en su asociación con una maqueta. Se puede realizar ya sea en cartón o en material más durable, como contrachapado - cualquier tipo de aglomerado (este caso triplay). Se pega sobre esta base una gráfica que se describe a continuación: el trazo comprende un círculo dividido en grados dobles. Permite su orientación con base en un norte geográfico o magnético, para hacer posible la lectura de ángulos acimutales. La diferencia entre estos nortes aparece en la gráfica de isógonas publicada en el Atlas Solar de la República Mexicana. Esta gráfica incluye unos círculos concéntricos que registran los ángulos de altura del sol a partir de la proyección en sombra de un gnomo ubicado en el centro del círculo, perpendicular al plano de la gráfica.

La Figura 2 nos muestra el Gnomo horizontal donde indica los horarios de la Ciudad de Poza Rica de Hidalgo, Veracruz. Obtenida desde el programa Shadows Pro.

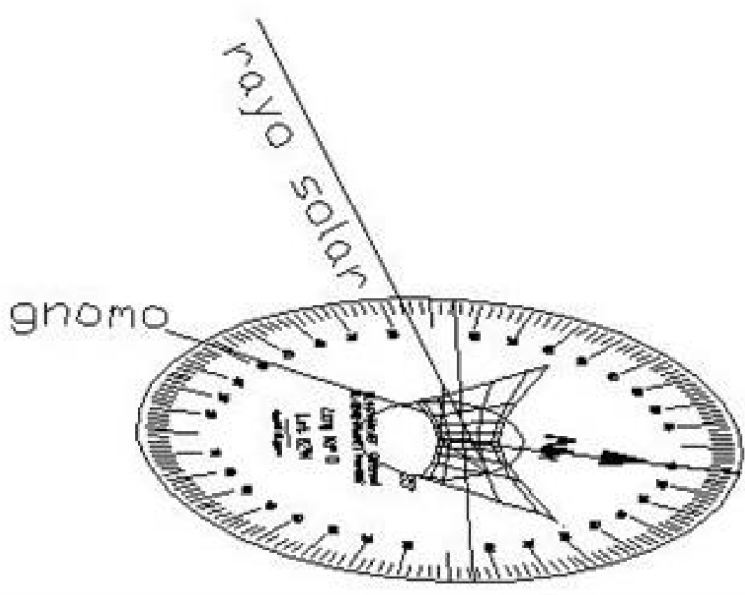

Figura 1. Funcionamiento correcto del Heliodón mediante la gráfica.

Fuente: Tamiset, Tirado y Gámez (2013)

Sobre la base plana se colocan la maqueta y el Heliodón asegurando un paralelismo entre los nortes (sea magnético o geográfico) de ambos. Frente al sol se inclina la base hasta llevar la extremidad de la sombra del gnomo en coincidencia con el punto de la gráfica de la fecha y hora para las cuales queremos visualizar la iluminación solar de la maqueta con sus efectos de sombra (Figura 2). De las fechas tenemos un solo día de cada mes: los 21; y de las horas una variación de hora en hora. Como resultado podemos simular la variación de la posición del sol 9 horas de un día, el 21 de cada mes. Son así $9 \times 7=63$ posiciones. 


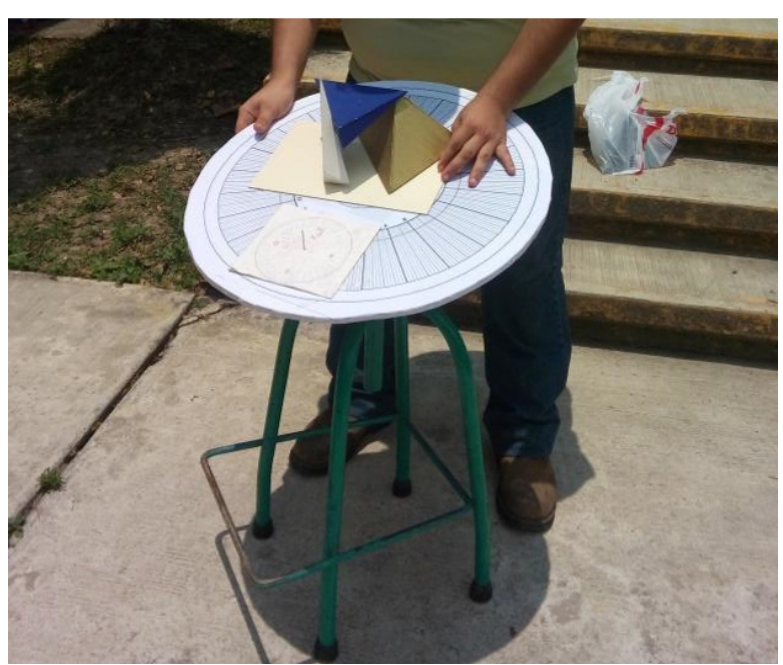

Figura 2. Demostración del uso correcto del prototipo. Fuente: Los autores, 2021.

Este Heliodón permite obtener una información de asoleamiento suficientemente precisa para la toma de decisión en el diseño arquitectónico e instalaciones solares. Además de permitir la visualización tridimensional de los efectos de sombras lee simultáneamente las coordenadas solares del momento escogido. V. La propuesta es que la bóveda celeste tenga movimiento, representando las horas y los grados en los que proyectan la sombra. De acuerdo con este factor se propuso como herramienta indispensable una Figura esférica que está adosada por una barra de acero, esta esfera genera una articulación y provoca un movimiento libre. Los materiales y procedimientos utilizados para este prototipo fueron:

Madera: se realizó un círculo de triplay de $12 \mathrm{~mm}$ de espesor y de $60 \mathrm{~cm}$ de diámetro para representar la bóveda celeste.

Esfera de anclaje para autos: esta esfera de acero está adosada a un tubo de 2", este instrumento será necesario para poder anclar al plato de madera y a la base de modo que articulará la bóveda celeste ejemplificando una rótula de movimiento.

Tareas complementarias de herrería (anclaje de bóveda celeste a la esfera, alargamiento de tubo a la esfera, ajustador lateral), carpintería (base de heliodon) y desbaste base de torno (desgaste de tubo, manipulación modelística de la esfera).
Experimentación Virtual. El uso del software Shadows Pro: Por medio de este software podemos analizar la carta solar de la ciudad de Poza Rica de Hidalgo (lugar donde se harán las pruebas), así como las diferentes proyecciones de mapas de cuadrantes solares como el gnomo horizontal utilizado (cuadrante ecuatorial); este programa también nos facilita gráficas solares con precisión de tiempo para poder apreciar gráficas de índice de iluminación, círculo de azimuts con respecto a la latitud y longitud de la región donde nos encontremos. También, por medio del software de Autodesk Ecotec Analysis (Figura 3), es posible realizar este tipo de ejercicios a través de un modelo tridimensional indicando fecha y región donde nos encontremos.

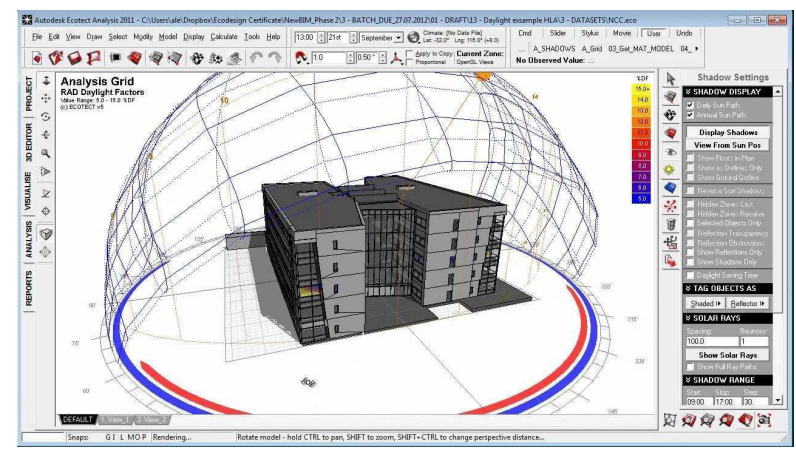

Figura 3. Software de Autodesk: Ecotec Systems Fuente: Los autores, 2021.

Recurrimos a la experimentación manual, ubicando las coordenadas en el mapa solar por medio de la aplicación Sun Surveyor (Figura 4) a través de un dispositivo móvil, ya que mediante la ubicación en la que nos encontremos se proyectará una brújula y representación solar dependiendo de la fecha y la hora.

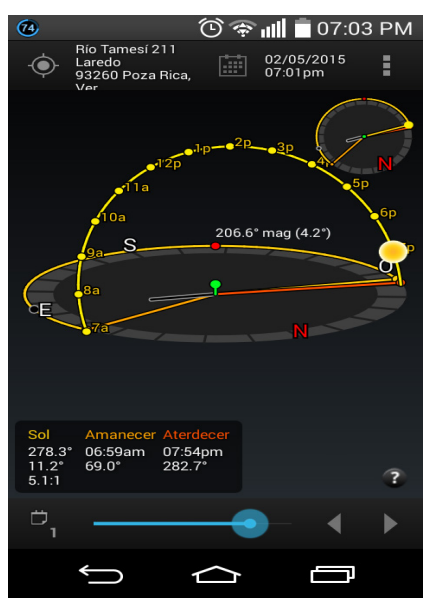

Figura 4. Software Sun Sevoyer Fuente: Los autores, 2021. 
El Uso de la Carta Solar. La carta solar estereográfica es una representación en planta del recorrido solar que permite una lectura bastante directa de la posición del sol, muy útil para el estudio de espacios abiertos exteriores, permite sacar de manera manual, eficiente y exacta la posición del sol respecto a la fecha y horarios, también podemos saber la distancia en grados del azimut. Una vez teniendo clara la teoría del funcionamiento del Heliodón procedimos a la construcción del prototipo siguiendo los siguientes pasos:

\section{Proceso Constructivo.}

1. Representación de la esfera relacionada con el plano y puesta en la base (Figura 5).

2. Esfera que dará movilidad a la bóveda celeste para la manipulación al representar la proyección de sombras (Figura 6).

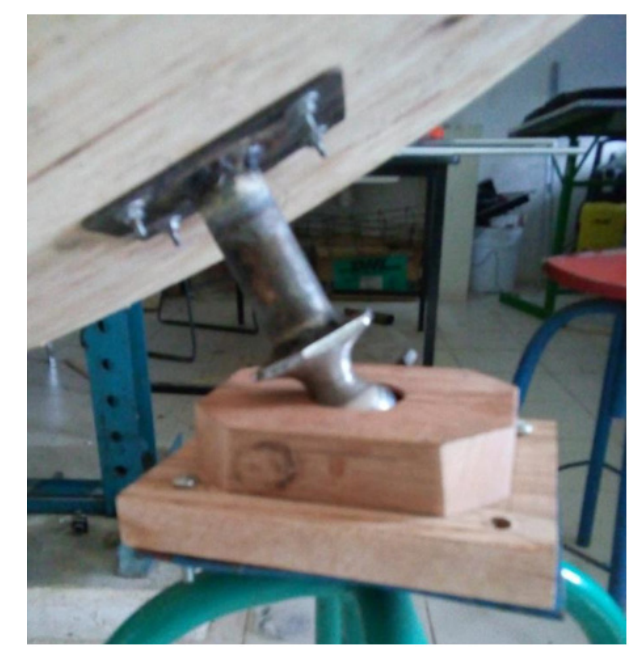

Figura 5. Colocación de esfera con la base. Fuente: Los autores, 2021

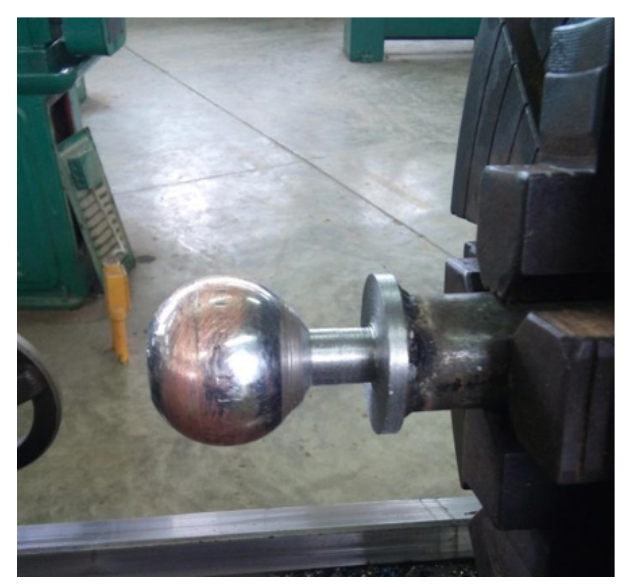

Figura 6. Esfera de acero para articulación Fuente: Los autores, 2021
3. Reloj solar con coordenadas latitud y longitud de la ciudad de Poza Rica Veracruz (Figura 7).

4. Heliodón anclado a su Base (Figura 8).

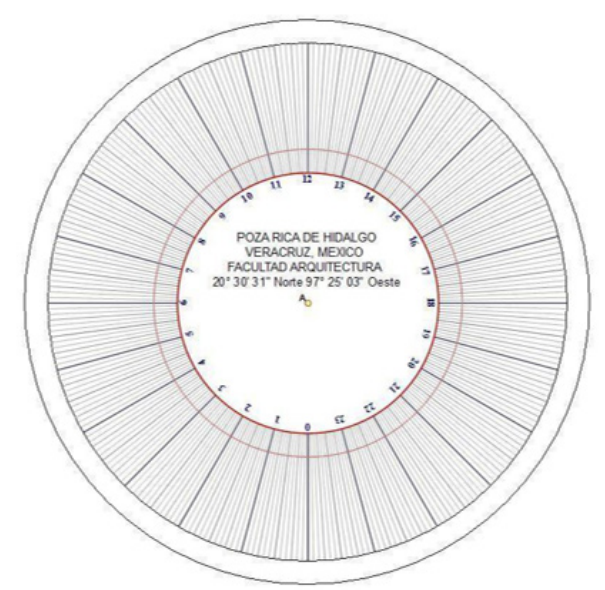

Figura 7. Gráfica para utilizar.

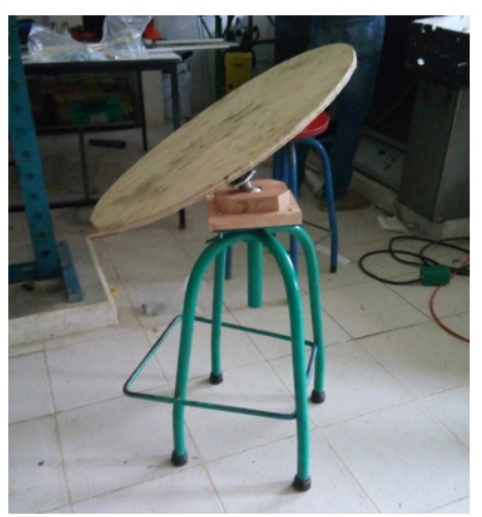

Figura 8. Ensamblaje completo Fuente: Los autores, 2021.

Este Heliodón sirve para la proyección de sombra proyectada en diferentes estaciones del año y horarios. Se procede a realizar pruebas de aplicación y experimentación, utilizando el prototipo, con la finalidad de verificar la proyección de las sombras en maquetas a escala (Figuras 9 y 10). 


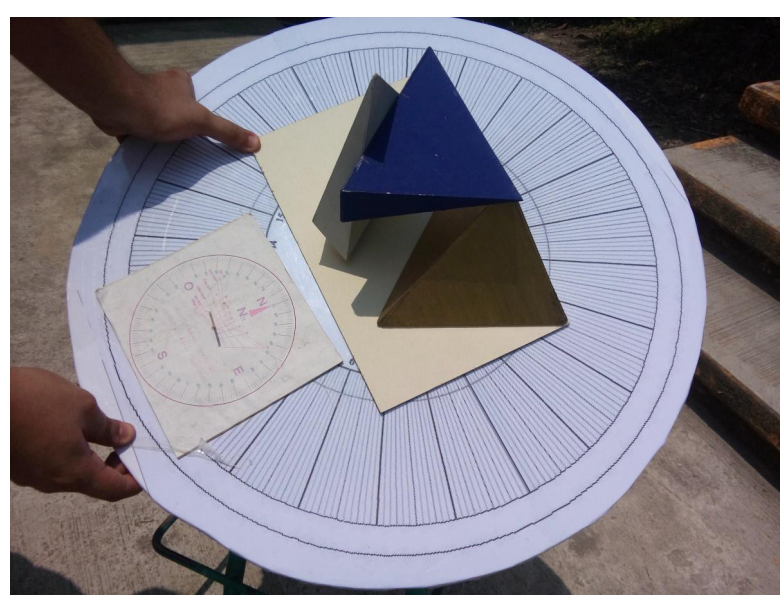

Figura 9. Prueba en el Heliodón donde se proyecta la sombra en el modelo en la fecha 21 de diciembre a las 16:00 hrs.

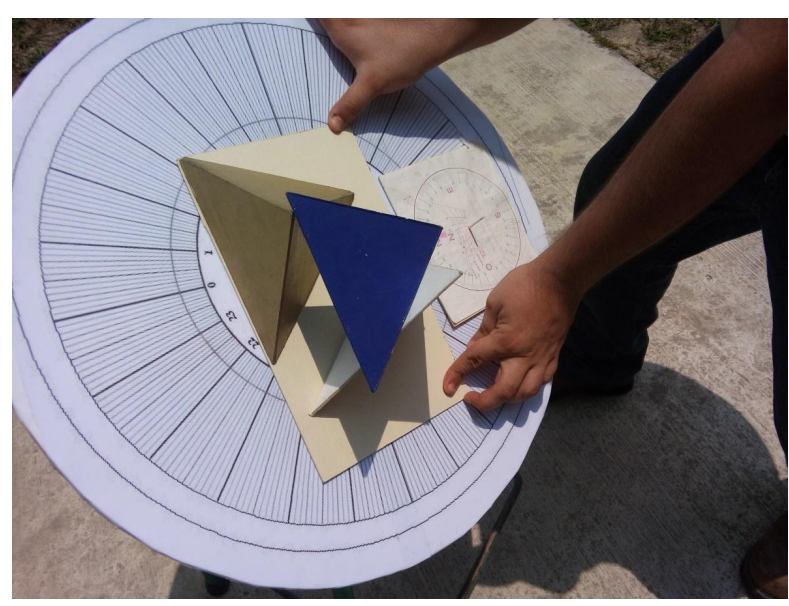

Figura 10. Prueba 21 de noviembre/enero a las 09:00 hrs. Fuente: Los autores, 2021

Segundo prototipo. Debido a que la gran mayoría de dispositivos existentes de Heliodón están orientados hacia 2 vertientes específicas las cuales son:

1. Dispositivos didácticos de aproximación de datos (los cuales están construidos y diseñados con el objetivo de generar las sombras de un proyecto en una ubicación específica y no son capaces de adaptarse a las diversas estaciones del año ni a la ubicación de otras partes del mundo).

2. Dispositivos Profesionales Especializados (estos son capaces de generar las proyecciones solares en casi cualquier parte del mundo a cualquier hora y fecha del año, pero utilizan un sistema computarizado muy complejo o requieren una fabricación muy costosa debido al tamaño, los materiales y accesorios que utiliza).
Fue necesario desarrollar un prototipo mixto, es decir, que cumpliera con la mayoría de tareas que puede realizar un dispositivo profesional pero sin la complejidad de fabricación y de bajo costo, además de que también cumpliera con la funcionalidad en su diseño y que tuviera cierta estética, ya que a diferencia del primer prototipo, se desarrolló un dispositivo más eficaz y más fácil de utilizar.

El primer aspecto a tener en cuenta fue el material de construcción: se debía elegir un elemento que fuera resistente, moldeable, ligero, barato y fácil de transportar, por lo que se eligió el MDF(medium density fibreboard) el cual es una fibra de madera de densidad media que es un material muy cómodo de utilizar y da un acabado de madera muy elegante. Posteriormente, se realizó el diseño del dispositivo, el cual se decidió que la base principal fuera un círculo de $1.20 \mathrm{~m}$ de diámetro con un plato central giratorio para la ubicación del norte; la base posee 2 anclajes principales los cuales son para fijar los arcos estacionales y a su vez estos arcos pequeños de $15 \mathrm{~cm}$ sostienen el arco principal de trayectoria solar que abarca toda la base.

El arco principal se diseñó con una hendidura en uno de sus lados para la colocación de un carrito a modo de riel el cual sostiene la lámpara y permite que la misma se desplace por todo el arco. Hay que mencionar que el arco principal está dividido en 11 partes iguales, debido a que el sol da sus primeros rayos de luz a las 7 am y se oculta a las $17 \mathrm{hrs}$, teniendo en realidad 11 horas de luz la mayoría del año; los arcos pequeños que están anclados a la base están divididos en 6 , ya que son 3 épocas del año las que se consideran: el solsticio de invierno, equinoccio de primavera y solsticio de verano. $Y$ dependiendo de la ubicación geográfica del proyecto ya sea si está ubicado en el hemisferio norte o sur es como se da la inclinación del arco principal, es decir, cada hemisferio (norte y sur) tiene su nomenclatura dividida en 3.

El plato base tiene 2 nomenclaturas: la primera es la rosa de los vientos para ubicar correctamente el norte del proyecto; y la segunda es una cuadrícula que representa la latitud y longitud de las coordenadas geográficas del proyecto, de modo que están plasmadas como un plano cartesiano. De este modo lo único que hay que hacer es ubicar la maqueta o modelo $3 \mathrm{~d}$ sobre el centro del punto más aproximado a las coordenadas de ubicación. La estructura está soportada por una base compuesta bajo el arco y permite el giro del 
mismo para simular las variaciones de solsticios y equinoccios a lo largo del año; también permite el giro del plato central para ajustar el norte según la ubicación del predio o edificio a ubicar.

Materiales y Herramientas para la Elaboración del Prototipo. Para el diseño del prototipo utilizamos el programa de dibujo arquitectónico AutoCAD 2019. Todas las piezas así como sus componentes se dibujaron y se verificaron antes de mandarlo a corte láser. Para la construcción del prototipo, se utilizó el MDF debido al costo relativamente bajo y la facilidad de corte; fueron necesarias 10 placas completas de MDF de $90 \times 60 \mathrm{~cm}$ y $6 \mathrm{~mm}$ de grosor para hacer todas las piezas y una lámpara alimentada por una batería de $9 v$, la cual desempeña el papel de representación de la luz solar y una maqueta escala 1:100 de una vivienda.

Otros materiales:

Pegafuerte (marca parisina)

Tuercas y rondanas de acero $3 / 8^{\prime \prime}$

Silicón frío para unir las piezas

Proceso de Diseño y Construcción. Una vez concluido el proceso de diseño y de fabricación de las piezas, se procede al ensamblaje del dispositivo, ya que previamente el diseño en digital se mandó a un estudio de corte láser para que el corte de las piezas fuese más preciso, limpio y rápido. Al recibir todas las piezas, nuestra tarea fue armar todos los elementos del Heliodón y hacer las respectivas pruebas de funcionamiento siguiendo los siguientes procedimientos:

I. Dibujo de pieza por pieza en AutoCAD, teniendo claro el diámetro del plato (base principal) de 1.20 mts y la altura del arco solar de $60 \mathrm{~cm}$ pudimos diseñar los elementos de la estructura, tanto de la base como el funcionamiento de los accesorios; la lámpara, el plato giratorio y las nomenclaturas de los arcos y de la base (Figura 11 y12).

II. Traslado de material: Al término del corte láser, proseguimos con el transporte de las placas de MDF y verificamos que estuvieran ordenadas y completas; en este caso fueron necesarias 10 placas de MDF de 90×60 $\mathrm{cm}$ dentro de las cuales contenían todas las piezas para el armado del Heliodón.

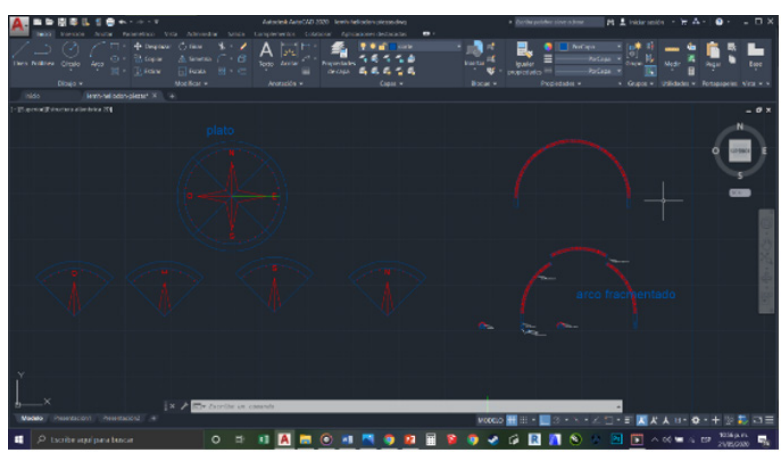

Figura 11. Diseño del arco solar acorde con los ángulos del sol en las horas del día

Fuente: Los autores, 2021.

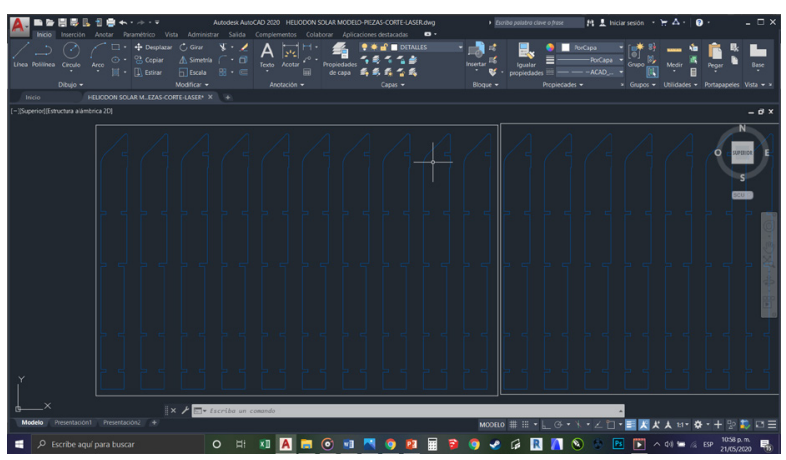

Figura 12. Diseño de piezas para soporte de la base Fuente: Los autores, 2021

III. Despiece de todos los elementos y orden por secciones para un manejo más eficiente y un armado más rápido. En este paso fue necesario sacar todas las piezas de las placas y separarlas por grupos para empezar a armar cada elemento por separado.

IV. Armado de la base circular y del plato central. En esta sección procedimos con el ensamblado de la base principal y la colocación del rodamiento para el plato base (Figura 13).

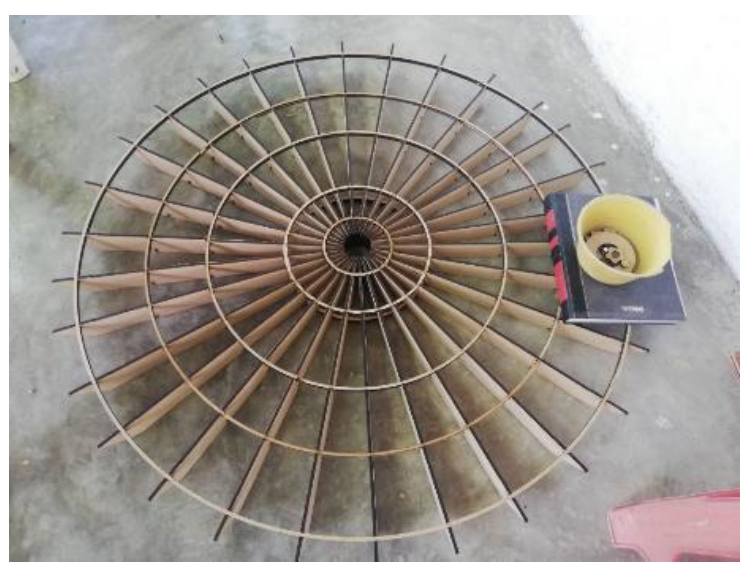

Figura 13. Pegado y armado de la base de apoyo del Heliodón. Fuente: Los autores, 2021. 
Armado de arcos y colocación sobre el plato central. Una vez teniendo la base, continuamos con la colocación de los arcos estacionales los cuales sirvieron de anclaje para el arco solar principal (Figura $14,15$ y 16$)$.

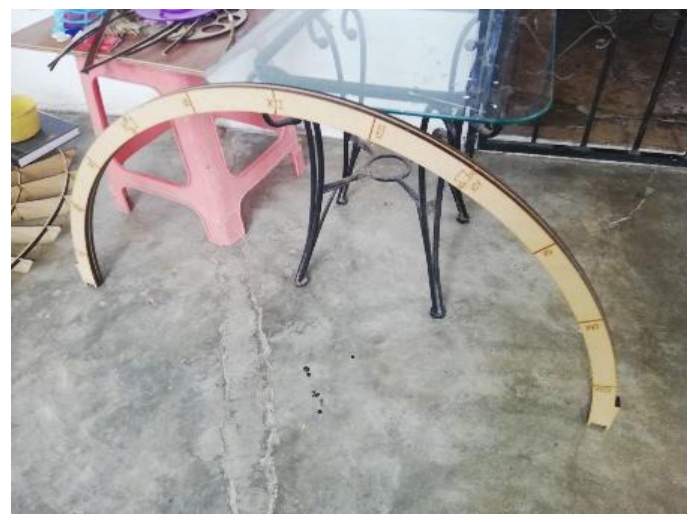

Figura 14. Pegado y armado de arco solar. Fuente: Los autores, 2021.

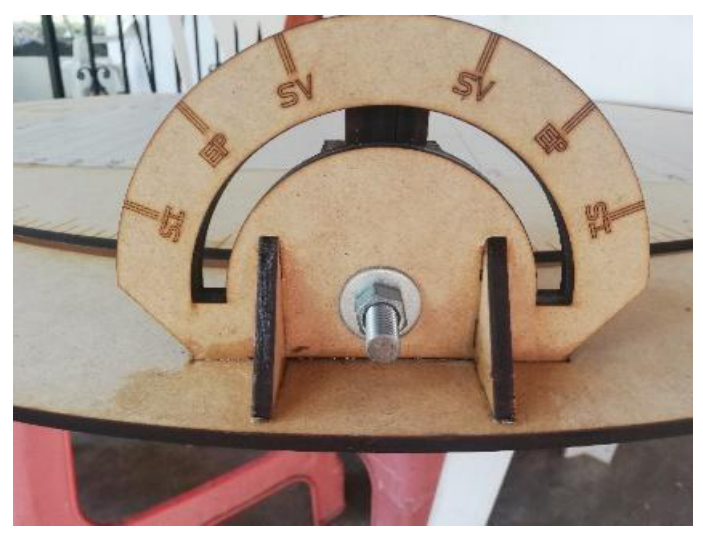

Figura 15. Pegado y armado de arco medidor de solsticios y montaje sobre el plato central.

Fuente: Los autores, 2021.

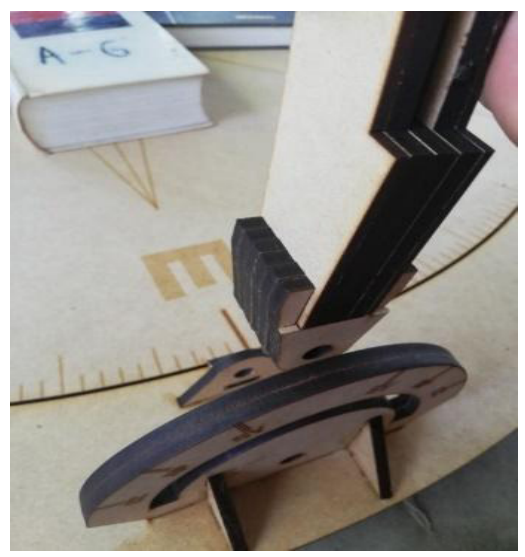

Figura 16. Montaje de arco solar sobre el plato central y unión con el arco medidor.

Fuente: Los autores, 2021.
VI. Armado y pegado de dispositivo portador de la lámpara y montaje de la lámpara sobre el arco solar. Para este punto ya están colocados los elementos principales y solo resta ensamblar los accesorios al arco solar, en este caso el carrito con los rodamientos y la lámpara (Figura 17, 18 y 19).

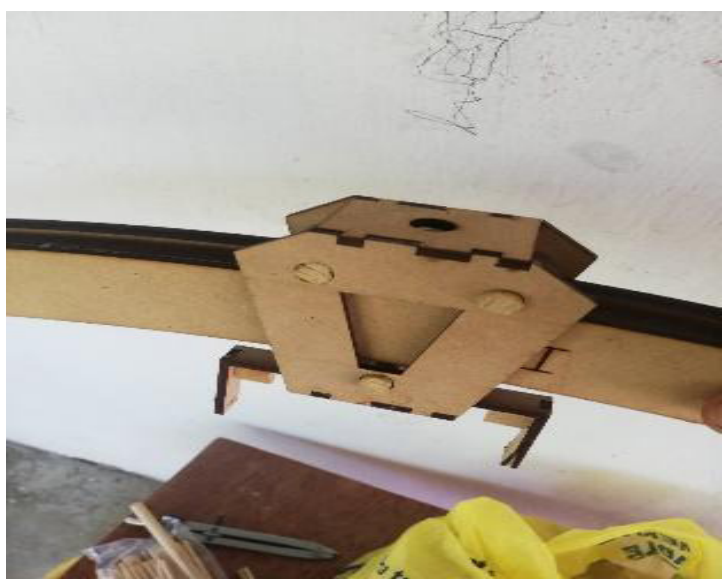

Figura 17. Prueba de rodamiento sobre el arco solar. Fuente: Los autores, 2021.

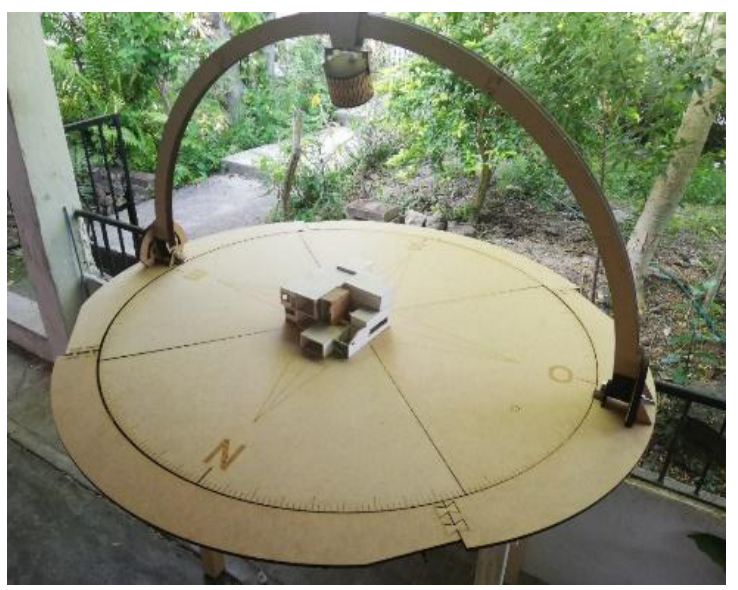

Figura 18. Armado finalizado del prototipo Fuente: Los autores, 2021

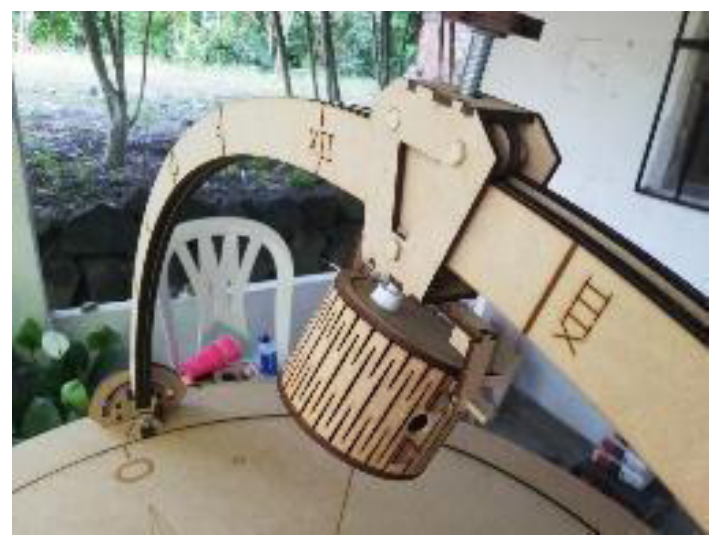

Figura 19. Montaje de lámpara en el arco principal Fuente: Los autores, 2021. 
VII. Por último la colocación de retícula de latitud y longitud sobre el plato central. El propósito de esta es proporcionar un acercamiento a las coordenadas de ubicación geográficas, aunque no proporciona datos exactos brinda un buen acercamiento (Figura 20, 22 y 23).

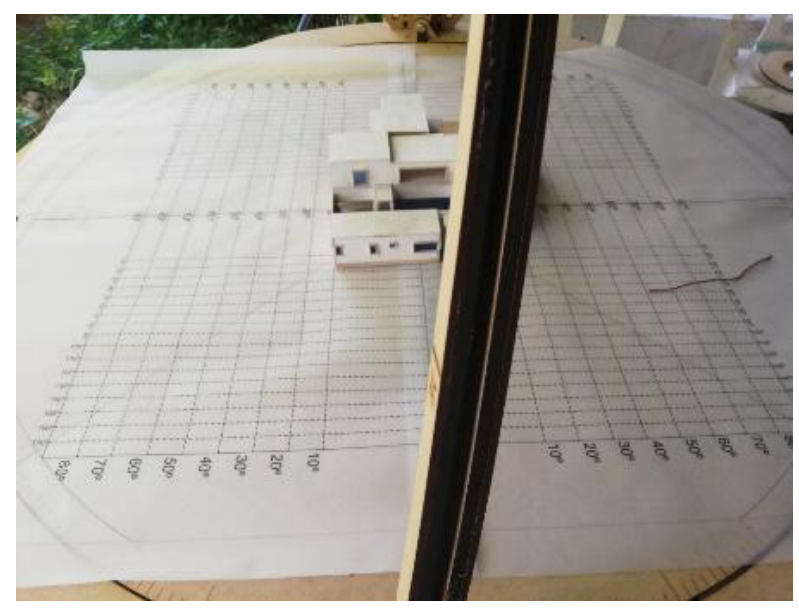

Figura 20. Trazado de posición y pegado de la retícula sobre el plato central.

Fuente: Los autores, 2021.

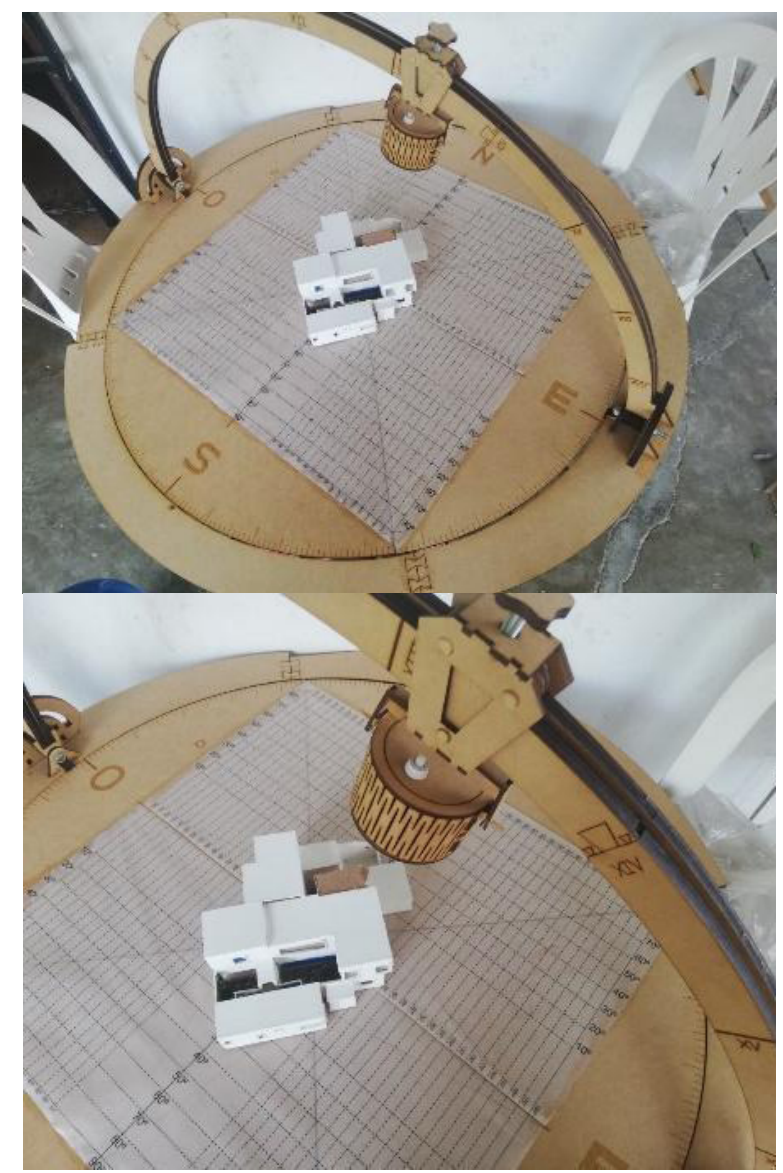

Figura 21. Prototipo listo y terminado.

Fuente: Los autores, 2021.
VIII. Pruebas de funcionamiento. Una vez terminado el ensamblaje del prototipo se verificó el correcto funcionamiento del Heliodón simulando las coordenadas de ubicación geográfica de la ciudad de Poza Rica $\left(20^{\circ} 32^{\prime \prime}\right.$ latitud norte y $97^{\circ} 27^{\prime \prime}$ longitud oeste), con fecha y hora específicas y con su debida orientación del norte (Figura 23 y 24).

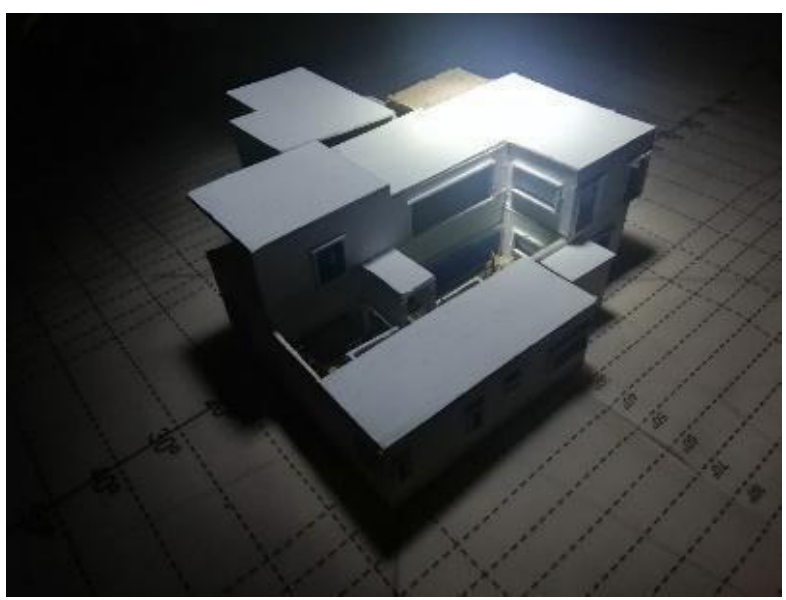

Figura 23. Segundo ensayo de funcionamiento, ubicación: Poza Rica, 1 PM, Solsticio de Verano. Fuente: Los autores, 2021.

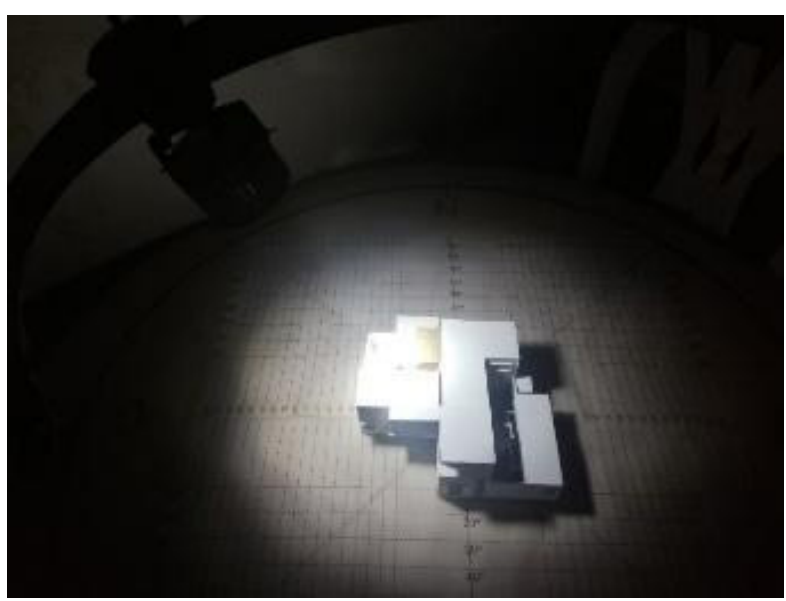

Figura 24. Segundo ensayo de funcionamiento, ubicación: Poza Rica,10 AM, Solsticio de Verano.

Fuente: Los autores, 2021.

Ejercicio de orientación de una edificación conociendo los datos del Heliodón. El acceso solar es algo que todo proyecto debe aprovechar $\mathrm{y}$ es de suma importancia para poder instalar sistemas captadores de energía solar y también para adaptar los espacios a la iluminación natural (Medina y Samper, 2016). 
La orientación es uno de los principales factores que un Arquitecto debe tener en cuenta al momento de diseñar una edificación los principales motivos son el ahorro de iluminación y climatización artificial hasta un $70 \%$. Se darán las orientaciones recomendadas, por ejemplo: al referirse a "orientación norte" esto quiere decir que la fachada principal del proyecto estará ubicada hacia esa dirección.

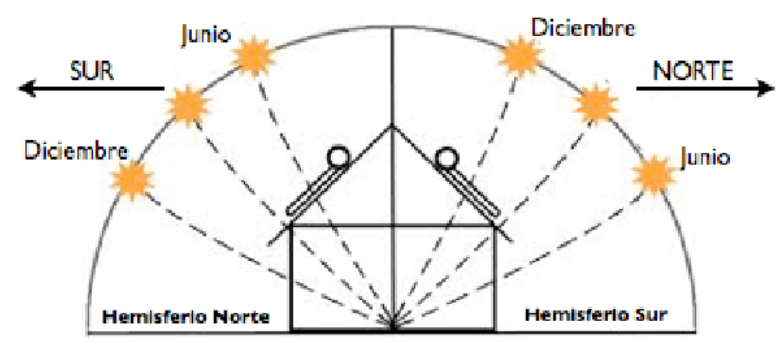

Figura 25. Diferencia del Impacto solar entre el hemisferio norte y sur.

Fuente: Romero (2017).

Hablando sobre el hemisferio norte:

La Orientación Sur: Es la mejor opción para climas semicálidos, templados y fríos ya que la estación invernal es más larga y recibe el impacto de radiación solar todo el año lo que permite aprovechar al máximo la iluminación natural. Sin embargo, para climas cálidos es necesario colocar un alero que disminuya el exceso de radiación solar durante el verano; en cuanto a lo arquitectónico es ideal para estancias grandes y dormitorios ya que por la noche se libera el calor acumulado durante el día.

La Orientación Sureste: Sería la segunda mejor orientación posible ya que en invierno recibe toda la radiación solar, pero en verano, a partir del mediodía, el impacto solar es menor, en zonas muy calurosas es más factible que la orientación sur.

La Orientación Este: Si elegimos esta orientación en zonas invernales intensas tendremos viviendas cálidas por las mañanas, pero noches frías por lo que la radiación e iluminación solar acumulada en las mañanas se dispersa a lo largo del día hasta llegar la tarde. A esta hora puede ser algo molesto el sol ya que el ángulo de incidencia es muy inclinado sobretodo en invierno. En este caso, si queremos dormitorios frescos por la noche conviene orientarlos hacia esta dirección.
La Orientación Noreste: Esta orientación es ideal para zonas de uso menor como garajes o almacenes ya que durante el invierno no recibe impacto solar directo en todo el día y en verano sólo por la mañana pues el sol sale por el sureste. La Orientación Norte: La única que recibe un impacto de iluminación uniforme durante todo el día, pero la mayor cantidad de radiación durante la mañana y tarde de verano ya que en invierno recibe una cantidad mínima de radiación directa. Es una muy buena opción para espacios de oficinas y despachos y también para garajes y cuartos de máquinas.

La Orientación Noroeste: Similar a la noreste, en este caso la incidencia solar en verano se recibe unas pocas horas durante la tarde y en invierno está ausente durante todo el día.

La Orientación Oeste: En situaciones de climas muy calurosos lo recomendable es no colocar ventanas hacia esta dirección o utilizar remetimientos y persianas para disminuir el impacto de radiación solar, ya que, de lo contrario, se recibe un exceso de la misma desde el mediodía hasta el anochecer y puede provocar un recalentamiento incómodo en las zonas ubicadas hacia esta orientación, aunque es ideal para zonas de fríos intensos.

La Orientación Suroeste: En este caso la radiación directa se recibe a partir del mediodía en verano e invierno y en climas cálidos se puede generar un sobrecalentamiento después del mediodía si no se toma en cuenta, por lo que es una orientación más adecuada a climas fríos y templados.

Se puede afirmar que en climas calurosos, en lo posible evitar utilizar la orientación sur ya que en verano es la que más sufre de incidencia solar, en estos casos una buena alternativa para mitigar una mala orientación es la utilización de sistemas pasivos como por ejemplo; toldos, aleros, linternillas, remetimientos o persianas. El uso de materiales de aislamiento térmico en los muros y cubiertas interiores también es una buena opción para conservar una temperatura interna confortable. En climas fríos y templados la orientación oeste es la mejor opción y en climas cálidos la mejor sería la orientación este, sin embargo hablando en términos generales en el hemisferio norte las mejores orientaciones son la este, sur y oeste, incluso en zonas calurosas (Romero, 2017).

Teniendo claro esta teoría, lo que ofrece el Heliodón es una vista espacial más realista del impacto solar sobre un proyecto, ya que de este modo podemos 
analizar la incidencia solar con detalle y generar una idea mucho más directa y precisa del correcto diseño, posición y tamaño de una ventana, tragaluz o remetimiento necesarios (Alonso Julio, 2017).

Una vez que revisamos la trayectoria solar y el impacto que tiene sobre el proyecto, considerando además la utilización de los materiales más adecuados para mitigar la incidencia extrema, podremos decir que desarrollamos el proyecto dentro de los parámetros básicos para el diseño bioclimático (Echeverri Montes, 2020).

Algo a tener en cuenta en el diseño de un proyecto arquitectónico es que la vegetación y las edificaciones cercanas pueden afectar o beneficiar la radiación solar que recibe nuestro proyecto (Sergi Gargallo, 2016).

La iluminación en el espacio arquitectónico. La orientación correcta se complementa con un correcto sistema de iluminación natural, ya que es un recurso que hay que gestionar coherentemente, porque es indispensable en el diseño arquitectónico llegando a desarrollar diversas técnicas para destacar tu estilo personal en los proyectos (Seguí, 2016).

Generalmente, podemos decir que las ventanas son el elemento principal por el cual suministramos iluminación natural al interior, pero la mayoría de las veces no consideramos el factor "acristalamiento" que se genera paralelamente con la inclusión de las ventanas. Un acristalamiento muy elevado puede provocar un calor extremo y deslumbramiento, pero uno muy bajo produce un nivel de iluminación pobre y carente de estética, es por ello que es de suma importancia lograr un equilibrio en el tamaño y posicionamiento de las ventanas en los proyectos que diseñemos (De los Reyes, 2016). Algunosfactoresimportantesquedebemosconsiderar para lograr una buena calidad de luz ambiental son: la orientación del edificio, el emplazamiento, la forma y dimensiones de los espacios y la distribución arquitectónica (Serrano, 2016).

Podemos controlar la luz natural con elementos arquitectónicos como muros cortina, aberturas en los muros, linternillas, tragaluz, apantallamientos, remetimientos de puertas y ventanas, etc. Todo con el fin de ajustar a nuestros gustos y necesidades la entrada de luz natural a nuestros espacios, los motivos principales para implementar estos sistemas pasivos son: ahorro de energía y confort del usuario (IDAE, 2005).

\section{DISCUSIÓN}

El Heliodón es una extraordinaria herramienta. Sin embargo, su versatilidad depende de su forma de construcción y del uso que se le dé; es por ello que muy pocas universidades e instituciones de investigación han construido o utilizan este dispositivo.

Como bien sabemos en Latinoamérica hay muchas limitantes en cuanto a su infraestructura y herramientas de trabajo para el desarrollo de prototipados especializados como el Heliodón, pero, a pesar de esto, se han realizado propuestas muy interesantes por estudiantes e investigadores, he aquí unos cuantos ejemplos a destacar (Figura 26-29):

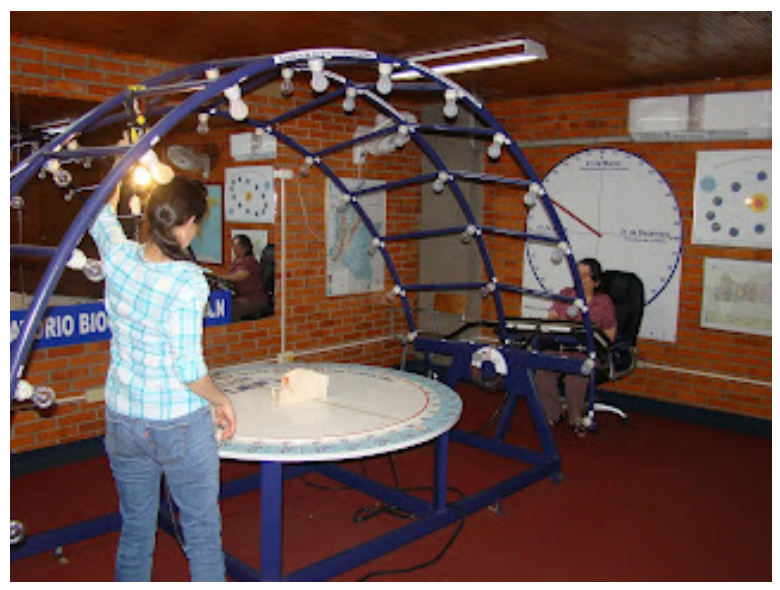

Figura 26. Heliodón hecho por estudiantes en la Universidad Antonio Nariño (Colombia).

Fuente: UAN Palmira (2016)

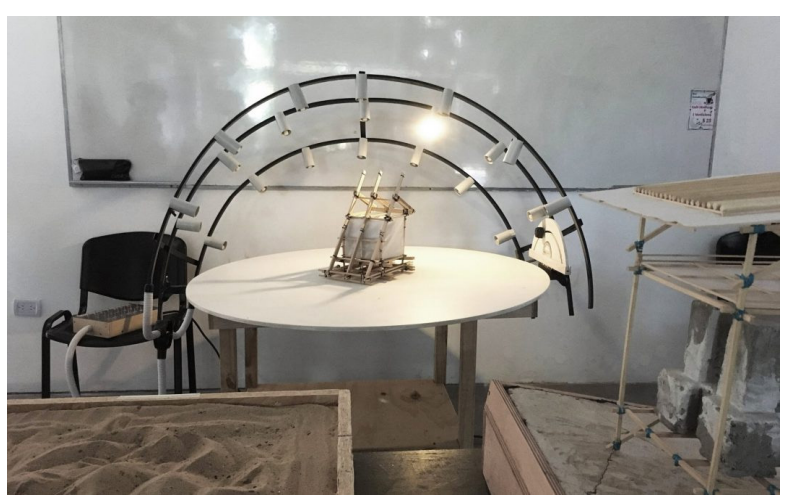

Figura 27. Construcción de un Heliodón por estudiantes de la UNSAM en Argentina.

Fuente: Heredia \& Orellano (2018). 


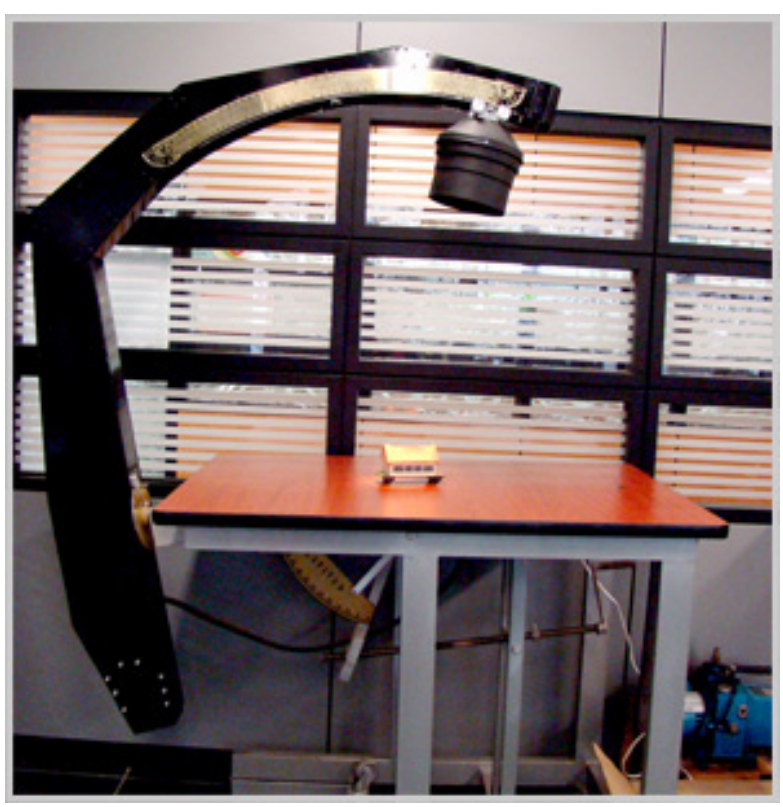

Figura 28. Heliodón mecánico construido en la UNAM Fuente: Salgado \& Morillón (2007).

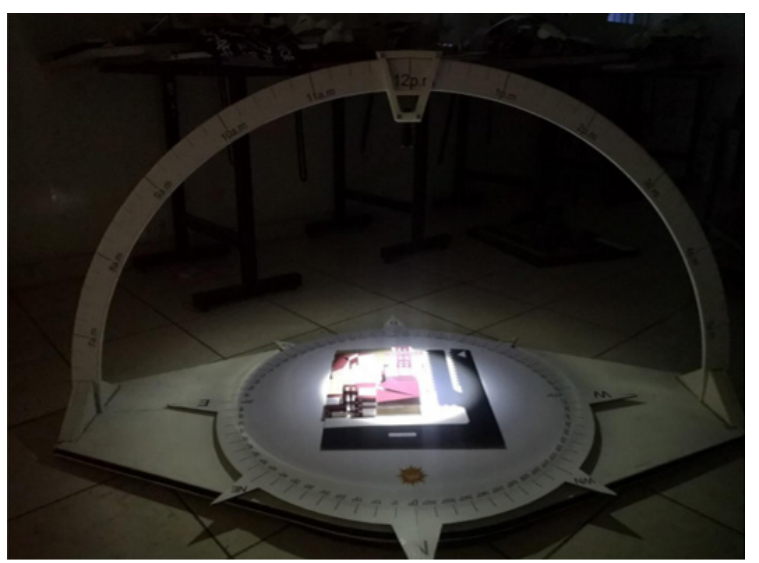

Figura 29. Construcción de un Heliodón Universidad Continental (Huancayo, Perú).

Fuente: Gómez \& Román (2020).

Como se puede apreciar, todos estos modelos de Heliodón fueron desarrollados con el objetivo de otorgarle al arquitecto una visión más realista del efecto solar sobre las edificaciones; sin embargo, cada modelo tiene sus puntos fuertes y débiles, pero todos tienen la versatilidad de poder interactuar fácilmente con la maqueta y con la posición de la luminaria. Lo cierto es que el software simulador de trayectorias solares ofrece resultados más precisos y de mejor calidad que un modelo análogo de un Heliodón, pero es más complejo de utilizar y sobretodo carece del carácter didáctico que nos brinda un dispositivo físico como el Heliodón, ya que el hecho de poder ver y palpar el comportamiento del sol ante un escenario real nos da la flexibilidad de ajustar el dispositivo de forma manual y sencilla sin depender de un software (Morató \& Fábregas, 2016).

Como en muchos campos de la ciencia, el software de simulación solar en Arquitectura está siendo más utilizado actualmente debido a la comodidad de visualizar estos datos en una pantalla, en vez de utilizar un dispositivo de gran tamaño. Sin embargo, esto no significa que el Heliodón quede descontinuado, sino al contrario, con una correcta construcción y equipamiento de accesorios modernos podemos generar una herramienta simuladora de trayectorias solares mejor que cualquier software, pero es conveniente seguir algunas recomendaciones de diseño y construcción:

Desarrollarlo y construirlo con materiales ligeros, resistentes y duraderos. 2. De un tamaño considerable para que las sombras se puedan apreciar mejor en maquetas de buen tamaño. 3. En un sitio donde no se tenga que mover (es decir lo más recomendable es que sea fijo y en un espacio oscuro). Y 4. Equiparlo con una luminaria de xenón que emita luz unidireccional (esto nos dará mayor potencia y mejor definición de sombras). $\mathrm{Si}$ seguimos estas recomendaciones básicas podemos ser capaces de generar un Heliodón completamente profesional, que nos dará resultados similares o incluso mejores que un software.

\section{CONCLUSIONES}

La construcción del primer prototipo tiene el objetivo de dar a conocer una alternativa muy minimalista y económica de un Heliodón solar; sin embargo, a pesar de que su efectividad y precisión son suficientes para entender el comportamiento de las trayectorias solares pero carece de practicidad en cuanto a su uso efectivo, por lo que el diseño del segundo prototipo compensa esos faltantes añadiendo elementos más prácticos y simples de utilizar logrando mejores resultados.

La aplicación y uso del Heliodón didáctico en diferentes universidades ha dado como resultado mayor conciencia en el futuro arquitecto, fomentando diseños más sustentables y se ha logrado entender de manera visual y tridimensional el camino del sol con sus efectos en los diferentes 
diseños que analizamos a lo largo de su preparación dándole un valor agregado a sus conocimientos ya que, en años anteriores y en la mayoría de las escuelas de Arquitectura, no está incluido como tema la geometría solar, por su complejidad, o está incluido de manera ambigua.

Hoy podríamos decir que con la ayuda del Heliodón didáctico dicha complejidad deja de serlo y nos capacita a comprender y diseñar de manera congruente a las exigencias de esta época que tiene como común denominador el respeto al medio ambiente. El prototipo realizado cumple con las funciones básicas de un Heliodón didáctico. Sin embargo, concluimos que, para un mejor funcionamiento y sobretodo más exacto, es necesario pulir ciertos detalles como el material de construcción, el cual debería de ser de aluminio ligero o plástico resistente para mayor durabilidad, una lámpara de mayor potencia y con adaptador a corriente alterna, es decir, con un enchufe para una alimentación constante de energía, un mecanismo computarizado de movimiento de la lámpara y del arco principal para proporcionar datos tridimensionales mucho más exactos. Estas son algunas mejoras que en el futuro se deben considerar, si el objetivo es hacer un Heliodón profesional. Sin embargo, el prototipo funciona y cumple con los objetivos establecidos inicialmente.

La incidencia solar requiere de un análisis específico. $\mathrm{Si}$ se ejecutan las técnicas adecuadas se pueden lograr resultados muy buenos y resolver problemas de exceso de calor, mala orientación o falta de iluminación natural, incluso en edificaciones existentes, razón por la cual un Heliodón solar es una herramienta de gran apoyo que nos puede proporcionar información muy valiosa sin necesidad de software BIM (Gómez, 2018). Pero, pese a todas estas consideraciones, el confort térmico (en especial en los sitios de trabajo) es difícil de lograr debido a sus ambientes agresivos y de alta concurrencia, por lo que, en estos casos, es necesario evaluar el ambiente térmico mediante criterios específicos como número de usuarios, maquinaria que genera calor, modelo de infraestructura del edificio y materiales de construcción, razón por la cual en este tipo de edificaciones de trabajo es necesario equiparse con elementos de acondicionamiento de aire que cumplan con las características técnicas y operativas del sitio de trabajo (Villamar \& Silva, 2015).

Como arquitectos recae mucho sobre nosotros la responsabilidad de encontrar soluciones a la gran contaminación que se genera al construir un proyecto $\mathrm{y}$ de entre todos los elementos contaminantes el sector energético es el más importante, por lo que una de las soluciones más utilizadas actualmente por los constructores es implementar sistemas que disminuyan el consumo energético lo máximo posible, de modo que la climatización e iluminación sean proporcionados naturalmente por el entorno, y la utilización de instalaciones de iluminación y climatización artificiales sea utilizada lo mínimo posible, Estas estrategias se conocen como sistemas pasivos y el estudio del impacto solar sobre un proyecto es clave para el correcto uso de este tipo de sistemas (De León, 2011).

\section{REFERENCIAS}

Almanza-Salgado, R., y Morillón-Gálvez, D. (2007), Heliodón mecánico construido en la UNAM, Web Instituto de Ingeniería UNAM. Recuperado de: http://www2.iingen.unam.mx/es-mx/Investigacion/Laboratorios/ Paginas/EnergiaRenovableyDisenoBioclimatico.aspx

Beckers, B. (2007). Interpretación geométrica de la luz del cielo en el proyecto de Arquitectura. Recuperado de: http://www.heliodon.net/downloads/Beckers 2007 Helio 002 es.pdf

Blog de Ciencias Sociales (2016). Traslación y rayos solares. Recuperado de: http://kiko-sociales.blogspot. com/2012/09/traslacion-y-rayos-solares.html

De León-Estrada, A.R. (2011). La luz solar en la Arquitectura, Universidad de San Carlos de Guatemala. Tesis grado en Arquitectura, p. 14-16. Recuperado de: http://biblioteca.usac.edu.gt/tesis/02/02 2944.pdf 
De los Reyes-Cruz, M.J. (2016). La iluminación natural difusa en el interior de los espacios arquitectónicos. Tesis de posgrado en Ciencias en Arquitectura y Urbanismo Instituto Politécnico Nacional, p. 5456. Recuperado de: https://tesis.ipn.mx/bitstream/handle/123456789/21544/Tesis\%20-\%20 Mar\%C3\%ADa\%20Jimena\%20de\%20los\%20Reyes\%20Cruz.pdf?sequence=1\&isAllowed=y

Echeverri-Montes, P. (2020). Importancia de la trayectoria solar en la Arquitectura. Recuperado de: https:// www.echeverrimontes.com/blog/importancia-de-la-trayectoria-solar-en-la-Arquitectura

Fragoso, U. (2020). La astronomía en las antiguas civilizaciones. Sólo es ciencia. Recuperado de: https:// soloesciencia.com/2020/04/13/la-astronomia-en-las-antiguas-civilizaciones/

Franco-Medina, R., y Bright-Samper, P (2016). Acceso solar en la Arquitectura y la ciudad. Aproximación histórica. Revista de Arquitectura, 8(2), p. 95-106. doi:10.14718/RevArq.2016.18.2.9

Galván, J.M. (2005). Heliodón: simulador de la trayectoria del Sol. Esencia espacio (1), 90- 91. Recuperado de: https://www.repositoriodigital.ipn.mx/bitstream/123456789/24827/1/25-Heliodon.pdf

Gómez, A. (2018). Sol y Arquitectura. Artículo web, Lima, Perú. Recuperado de: https://repositorio.urp.edu. pe/bitstream/handle/urp/1118/Alejandro\%20Gomez\%20\%282017\%29\%20Sol\%20y\%20Arqitectura. pdf? sequence $=1 \&$ is Allowed $=y$

Gómez, K.; y Román, N. (2020). Uso de la fabricación digital aplicada a la construcción de un Heliodón en la Escuela Académico Profesional de Arquitectura de la Universidad Continental Huancayo al 2019. Tesis pregrado en Arquitectura. Facultad de Ingeniería Universidad Continental, p. 25. Recuperado de: https://alicia.concytec.gob.pe/vufind/Record/UCON 00aa6fe73a449a9268e6c7be4748ef42/Details

Heredia, H. y Orellano, G. (2018), Construcción de un Heliodón por estudiantes de la UNSAM (Universidad Nacional de San Martín) en Argentina. Web UNSAM. Recuperado de: http://noticias.unsam.edu. ar/2018/11/14/estudiantes-del-instituto-de-Arquitectura-construyeron-un-heliodon/

Instituto para la Diversificación y Ahorro de la Energía (IDAE), (2005), Guía Técnica de Aprovechamiento de la luz natural en la iluminación de edificios, Madrid, ISBN: 84-86850-92-4, p. 13-14. Recuperado de: https:// www.idae.es/uploads/documentos/documentos 10055 GT aprovechamiento luz natural 05 $\underline{f 12 a e 5 a . p d f}$

Laboratorio de Bioclimática UAN Palmira (2016). Heliodón - Simulador de sol para proyectos arquitectónicos. Universidad Antonio Nariño (Colombia). Recuperado de: http://laboratoriodebioclimaticauanpalmira. blogspot.com/2016/06/heliodon-simulador-de-sol-para.html

Maristany, A. ; Barra S.; Nicasio C.; Bonafé S.; Herrán C.; Abadía L. (2007). Diseño, construcción y ajuste de un prototipo de simulador de la trayectoria solar. Recuperado de: https://1library.co/document/download/ yj8xd82q?page $=1$

Morató, M.; y Fábregas, F. (2016). Heliodón 2.0 Simulación Solar. Recuperado de: https://issuu.com/moimo/ docs/heliod $n$

Rodríguez, D.M. (2007). El juego de las geometrías bajo el sol. Tesis de maestría en Arquitectura medio ambiental. Fundación UPC. Barcelona. Recuperado de: http://www.heliodon.net/downloads/El juego de las geometrias bajo el sol.pdf

Romero, A.J. (2017). ¿Cuál es la mejor orientación para tu vivienda? Madrid, España, Recuperado de: https:// www.arrevol.com/blog/cual-es-la-mejor-orientacion-para-tu-vivienda-casa 
Seguí-Seguí, P. (2016). Iluminación natural en Arquitectura. Recuperado de: https://ovacen.com/iluminacionnatural-en-Arquitectura/

Gargallo, S. (2016). El impacto de la radiación solar sobre el edificio. Recuperado de: https://sgarq.com/ impacto-la-radiacion-solar-edificio/

Serrano-Yuste, P. (2016). Luz natural y su aprovechamiento en los edificios. Recuperado de: https://www. certificadosenergeticos.com/luz-natural-aprovechamiento-edificios\#comments

Tamiset, J.F.; Tirado, G.; y Gámez, J. (2013). Simulador de iluminación solar. En Madrigal, J. (Ed.) Simuladores didácticos para Arquitectura y Construcción. Recuperado De: http://evirtual.uaslp.mx/ Habitat/innobitat01/CAHS/SS\%20Arq\%20Arista/Publicaciones/Libros/Libros\%20Publicados/ Simuladores\%20did\%C3\%A1cticos.\%20MGJF.\%20AGGJ.pdf

Villamar-Santamaría, B.; y Silva-Alvarado, F. (2015). Análisis de la incidencia de la radiación solar y su influencia en el aumento de temperatura al interior de las aulas de clases del bloque $K$ de la universidad estatal de milagro provincia del guayas. Tesis de grado en Ingeniería Industrial, Universidad Estatal de Milagro, p. 14, Recuperado de: http://repositorio.unemi.edu.ec/bitstream/123456789/2617/1/AN\%C3\%81LISIS\%20 DE\%20LA\%20INCIDENCIA\%20DE\%20LA\%20RADIACI\%C3\%93N\%20SOLAR\%20Y\%20SU\%20 INFLUENCIA\%20EN\%20EL\%20AUMENTO\%20DE\%20TEMP.pdf 